\title{
Performance Comparison of Continuous-Time IFLF and LF Low-Pass All-Pole OTA-C Filters
}

\author{
H. W. Su* and Y. Sun ${ }^{* *}$ \\ Department of Electronic, Communication and Electrical Engineering, Faculty of Engineering and \\ Information Sciences, University of Hertfordshire, Hatfield, Herts, AL10 9AB, U.K. \\ "Tel: ++44 (0)1707 285082, Email: H.W.I.Su@herts.ac.uk \\ ** Tel: ++44 (0)1707 284196, Fax: ++44 (0)1707 284199, Email: Y.Sun $($ hherts.ac.uk
}

\begin{abstract}
This paper presents the performance comparison of two typical multiple loop feedback all-pole operational transconductance amplifier-capacitor (OTA-C) filters: Inverse-follow-the-leader-feedback (IFLF) and Leapfrog (LF) filters. Two fourth-order structures are analysed and compared in terms of sensitivity, maximum input signal, noise, and OTA nonideality effects on magnitude response. Simulation results obtained show that the LF structure is better than the IFLF structure in these four performances. The comparison is based on the realisation of the fourthorder Butterworth low-pass characteristic with $1 \mathrm{MHz}$ cutoff frequency.
\end{abstract}

\section{Introduction}

Multiple loop feedback (MLF) filters have started to draw attention from filter researchers and designers since the 1970's. Research has shown that MLF filters have lower passband magnitude sensitivity than cascade filters [1]. Also, such filters can realise functions with nonimaginary-axis zeros that ladder filters cannot achieve. However, these circuits are unsuitable for high frequency applications and fully integrated implementation since they are opamp-based and use resistors. OTA-C filters offer advantages over opamp-based filters in terms of design simplicity, high frequency capability, electronic tunability, monolithic integrability and reduced component count. Many OTA-C biquadratic filter structures offering variety of filtering characteristics have been generated, and their performances have been evaluated and compared. Recently a systematic method for structure generation and design of MLF OTA-C filters has been proposed, along with a number of interesting structures and easy-to-use design formulae [2-7]. The application of the proposed structures in computer hard disk drive systems was reported [8]. However, none of the reported literature has systematically investigated the performance comparisons of high-order MLF OTA-C filters in terms of sensitivity, maximum input signal, noise, and magnitude response. The aim of this paper is therefore to compare different MLF OTA-C filters in terms of the above performances. Our interest is in the most popular IFLF and LF filter structures.
The paper is laid out as follows; Section II presents the structures and design equations of the two filters. Analysis and comparison in terms of sensitivity, maximum input signal, noise, and magnitude response are given in Section III, IV, V, and VI respectively. The paper concludes with a brief summary in Section VII. Comparison results given in the paper should provide useful guides to filter designers in choosing the best structure in their filter design.

\section{Fourth-Order IFLF and LF Structures}

The canonical IFLF and LF OTA-C filter structures [6] are shown in Fig. 1 . With $\tau_{j}=C_{j} / g_{m j}$ denoting the time constant of integrator $j$, the transfer functions of the IFLF and LF structures can be derived as given in (1) and (2) respectively.

$$
\begin{gathered}
H(s)=\frac{1}{\tau_{1} \tau_{2} \tau_{3} \tau_{4} s^{4}+\tau_{1} \tau_{2} \tau_{3} s^{3}+\tau_{1} \tau_{2} s^{2}+\tau_{1} s+1} \\
H(s)=\frac{1}{\tau_{1} \tau_{2} \tau_{3} \tau_{4} s^{4}+\tau_{1} \tau_{2} \tau_{3} s^{3}+\left(\tau_{1} \tau_{2}+\tau_{1} \tau_{4}+\tau_{3} \tau_{4}\right) s^{2}+\left(\tau_{1}+\tau_{3}\right) s+1}
\end{gathered}
$$

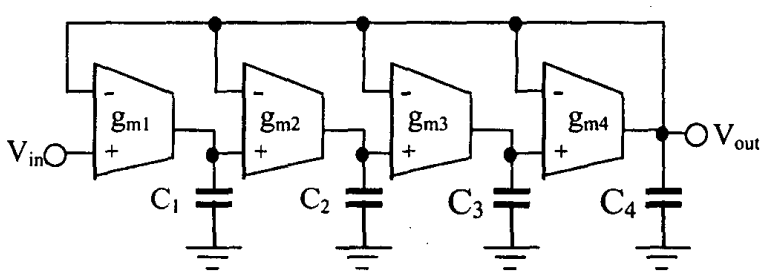

(a)

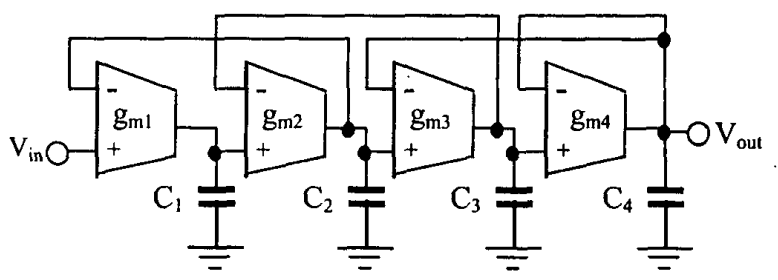

(b)

Fig.1. Canonical fourth-order (a) IFLF and (b) LF filter structures. 
The desired transfer function of the unity dc gain fourth-order all-pole low-pass filter is given by

$$
H(s)=\frac{1}{B_{4} s^{4}+B_{3} s^{3}+B_{2} s^{2}+B_{1} s+1}
$$

Compare (1) and (2) with (3) gives the design formulae for the IFLF and LF filters given in (4) and (5) respectively.

$$
\begin{gathered}
\tau_{1}=B_{1}, \quad \tau_{2}=\frac{B_{2}}{B_{1}}, \quad \tau_{3}=\frac{B_{3}}{B_{2}}, \quad \tau_{4}=\frac{B_{4}}{B_{3}} \\
\tau_{1}=B_{1}-\frac{B_{3}}{B}, \tau_{2}=\frac{B}{B_{1}-\frac{B_{3}}{B}}, \tau_{3}=\frac{B_{3}}{B}, \tau_{4}=\frac{B_{4}}{B_{3}}, B=B_{2}-\frac{B_{1} B_{4}}{B_{3}}(5)
\end{gathered}
$$

The normalised fourth-order Butterworth low-pass filter characteristic is given by

$$
\mathrm{H}(\mathrm{s})=\frac{1}{\mathrm{~s}^{4}+2.61313 \mathrm{~s}^{3}+3.41421 \mathrm{~s}^{2}+2.61313 \mathrm{~s}+1}
$$

When the filters realise the characteristic in (6), the values of $\tau_{j}$ for the IFLF and LF structures can be calculated using (4) and (5).

Note that both filter structures have the same number of differential OTAs and grounded capacitors. Thus, they have the same power consumption and chip area. But other performances will be different due to the difference in their feedback connections.

\section{Sensitivity Analysis and Comparison}

Sensitivity is one of the most important criteria in assessing the quality of filters. Thus, in the design of filters we need both to select low sensitivity realisations and reduce the sensitivities of the realisations we wish to use. This section compares the Schoeffler's multiparameter sensitivities of the fourth-order IFLF and LF filters.

The Schoeffler's multiparameter sensitivity is defined as [2]

$$
\mathrm{SS}=\sum_{i=1}^{1}\left|\mathrm{~S}_{i}^{\mathrm{H}(j \mathrm{j})}\right|^{2}
$$

where $S_{t}^{H(s)}$ are the sensitivities of transfer function $H(s)$ with respect to integration constants $\tau_{\mathbf{j}}$. Based on (1-5), the sensitivity functions of the IFLF and LF filters can be obtained. When both filters realise the normalised fourthorder characteristic in (6), using (7) their Schoeffler's multiparameter sensitivities can be calculated and are shown against the normalised frequency in Fig.2. It is clearly observed that the LF structure has lower sensitivity than the IFLF in the whole frequency range. In particular, the peak sensitivity of the IFLF is about 1.64 times that of the LF at the normalised frequency of about $1 \mathrm{rad} / \mathrm{s}$.

The worst case (WS) sensitivities of the filters are also computed. Based on [2], the worst case sensitivity in this case is defined as

$$
W S=\sum_{i=1}^{4}\left|S_{i}^{\mid N\left(j j_{0}\right)}\right| \mid
$$

The computed WS sensitivities agrees with the result obtained with the Schoeffler's multiparameter sensitivity

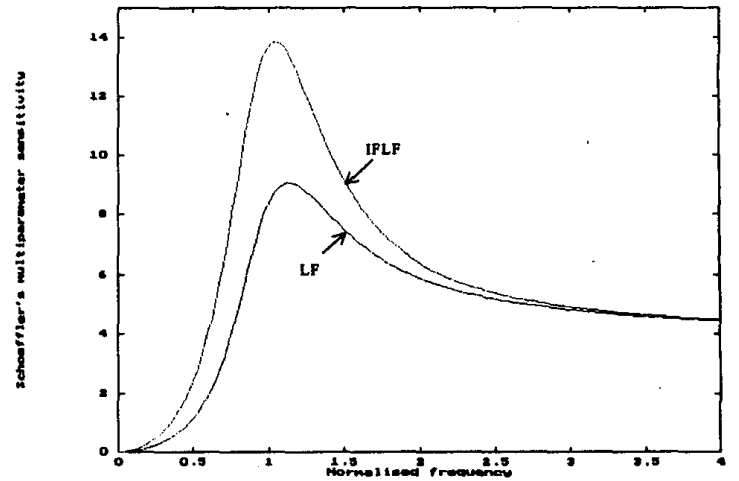

Fig.2. Comparison in terms of Schoeffler's sensitivity of fourth-order IFLF and LF filters.

comparison.

Thus, it is concluded that the LF filter is better than the IFLF filter in terms of sensitivity performance.

\section{Maximum Input Signal Analysis and Comparison}

OTAs have the limited differential input voltage swing required to maintain an acceptable degree of linearity. The maximum input voltage of a filter is related to its dynamic range (DR) which is defined as the ratio of the maximum signal level at the input or output to the minimum signal or noise level at the same point. Thus, when the maximum signal of the filter is known, then the filter's DR can be determined. The DR of a filter, given the transfer function, is dependent on the DR of the filter network elements and the filter network architecture. And the limited DR of the OTA is confined by the linear input range and noise level which restrict the DR of the filter [9]. This section will find the maximum input rologges of the IFLF and LF filters.

First consider the fourth-order IFLF filter structure. The transfer functions from the filter input to the differential inputs of integrators are obtained as

$$
\left.\begin{array}{c}
H_{1}^{\prime}(\mathrm{s})=1-\mathrm{H}_{4}(\mathrm{~s}), \mathrm{H}_{2}{ }^{\prime}(\mathrm{s})=\mathrm{H}_{1}(\mathrm{~s})-\mathrm{H}_{4}(\mathrm{~s}) \\
\mathrm{H}_{3}{ }^{\prime}(\mathrm{s})=\mathrm{H}_{2}(\mathrm{~s})-\mathrm{H}_{4}(\mathrm{~s}), \mathrm{H}_{4}^{\prime}(\mathrm{s})=\mathrm{H}_{3}(\mathrm{~s})-\mathrm{H}_{4}(\mathrm{~s})
\end{array}\right\}
$$

where $H_{j}^{\prime}(s)$ is defined as

$$
H_{j}^{\prime}(s)=\frac{V_{d j}}{V_{\text {in }}}, \quad j=1,2,3,4
$$

in which $\mathrm{V}_{\mathrm{dj}}$ is the differential input voltage of the $j$ th OTA and

$$
H_{j}(s)=\frac{V_{o j}}{V_{\text {in }}}, j=1,2,3,4
$$

in which $\mathrm{V}_{\mathrm{oj}}$ is the output voltage of the $j$ th integrator. So $\mathrm{H}_{\mathrm{j}}(\mathrm{s})$ is the transfer function from the filter input to the output of integrators. Using (4) and (9-11), the functions $H_{j}{ }^{\prime}(s)$ are obtained. We draw $\left|H_{j}^{\prime}(j \omega)\right|$ and from these 
curves we can identify the their maximum values as $\left|H_{2}{ }^{\prime}(j \omega)\right|_{\max } \approx 1.8,\left|H_{2}{ }^{\prime}(j \omega)\right|_{\max } \approx 1,\left|H_{3}{ }^{\prime}(j \omega)\right|_{\max } \approx 0.6$, and $\left|H^{\prime}{ }^{\prime}(j \omega)\right|_{\max } \approx 0.29$.

The maximum input voltages of the IFLF and LF filters are determined by [5]

$$
\begin{array}{r}
\left|V_{\text {in }}\right|_{\text {max }}=\min \left\{\frac{V_{T 1}}{\left|H_{1}^{\prime}(j \omega)\right|_{\text {max }}}, \frac{V_{T 2}}{\left|H_{2}^{\prime}(j \omega)\right|_{\text {max }}},\right. \\
\left.\frac{V_{T 3}}{\left|H_{3}^{\prime}(j \omega)\right|_{\max }}, \frac{V_{T_{1}}}{\left|H_{1}^{\prime}(j \omega)\right|_{\max }}\right\}
\end{array}
$$

where $\mathrm{V}_{\mathrm{Tj}}$ is the maximum linear differential input voltage of the $j$ OTA. Using (12), the maximum input voltage of the IFLF filter is determined as $0.56 \mathrm{~V}_{\mathrm{T}}$.

Now consider the fourth-order LF filter structure. The same set of transfer functions are obtained as

$$
\begin{gathered}
\mathrm{H}_{1}{ }^{\prime}(\mathrm{s})=1-\mathrm{H}_{2}(\mathrm{~s}), \mathrm{H}_{2}{ }^{\prime}(\mathrm{s})=\mathrm{H}_{1}(\mathrm{~s})-\mathrm{H}_{3}(\mathrm{~s}), \\
\mathrm{H}_{3}{ }^{\prime}(\mathrm{s})=\mathrm{H}_{2}(\mathrm{~s})-\mathrm{H}_{4}(\mathrm{~s}), \mathrm{H}_{4}{ }^{\prime}(\mathrm{s})=\mathrm{H}_{3}(\mathrm{~s})-\mathrm{H}_{4}(\mathrm{~s})
\end{gathered}
$$

Similarly, the magnitudes of these functions are drawn and their maximum values are identified as $\left|H_{1}{ }^{\prime}(j \omega)\right|_{\text {. }} \approx 1.6$, $\left|H_{2}{ }^{\prime}(j \omega)\right|_{-} \approx 1.42,\left|H_{,}{ }^{\prime}(j \omega)\right|_{\ldots} \approx 0.87$, and $\left|H_{4}{ }^{\prime}(j \omega)\right|_{\text {m. }} \approx 0.29$. The maximum input voltage of the LF filter is determined as $0.63 \mathrm{~V}_{\mathrm{T}}$ from (12). Note that in the above we assume $\mathrm{V}_{\mathrm{T}_{\mathrm{j}}}=$ $V_{T}$ for both structures.

The results reveal that the LF filter allows higher maximum input signal voltage than the IFLF filter.

\section{Noise Analysis and Comparison}

Noise performance of active filters is of considerable interest to filter designers and researchers since both the dynamic range of an active filter and the effectiveness of signals recovery from a noise background using an active filter are dependent on the output noise of the filter itself [10]. The output noise level of an active filter depends on its system transfer function, the quality of the passive and active devices, the general impedance level of the filter, and the topology of the filter [10]. In continuous-time OTA-C filters, noise is contributed by all OTAs, and interfering signals are coupled into the circuit in various ways. We can normally treat the overall effect as equivalent to injecting noise sources only at the inputs of integrators. In practice, it is usually reasonable to assume that noise sources are uncorrelated with one another.

Assume that the input noise signals all have white power spectral density and equal to $\mathrm{N}_{i}{ }^{2}$. Then the total output noise power spectral density (PSD) of the IFLF and LF filters is given by [11]

$$
\operatorname{PSD}_{w 0}(\omega)=N_{1}^{2} \sum_{k=1}^{4}\left|G_{k}(j \omega)\right|^{2}
$$

where $G_{k}(s)$ are the intermediate transfer functions from integrator inputs to the filter output. This section will find the output PSD functions of the fourth-order IFLF and LF structures.

From the definition of $G_{k}(s)$ given above, we can write

$$
G_{k}(s)=\frac{V_{\text {out }}}{V_{i k}}, \quad k=1,2,3,4
$$

Using (14) and (15), we get the output PSD functions $\operatorname{PSD}_{\text {no }}(\omega) / \mathrm{N}_{\mathrm{i}}^{2}$ which are shown in Fig.3. From the two curves, the output PSD of the IFLF configuration is lower than that of the LF for $0<\omega \leq 0.4 \mathrm{rad} / \mathrm{s}$, but thereafter the opposite situation occurs. In particular, the PSD of the IFLF structure is very much higher than that of the LF at $\omega=1 \mathrm{rad} / \mathrm{s}$ and in the region close to this frequency. Thus, we conclude that the PSD performance of the LF filter is much better than the IFLF filter over wide frequency range.

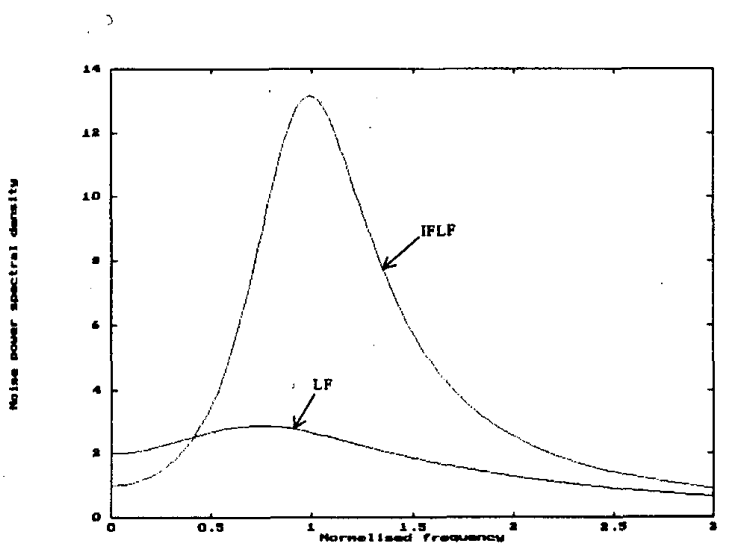

Fig.3. Comparison of noise performance of fourth-order IFLF and LF filters.

To further confirm the conclusion based on the PSD comparison, the output noise powers of the filters are computed using Simpson's $1 / 3$ rule. The output noise powers $(0 \leq \omega \leq 3)$ are computed as $P_{n o} / N_{i}{ }^{2}=22.53 \mathrm{~dB}$ and $\mathrm{P}_{\mathrm{no}} / \mathrm{N}_{\mathrm{i}}{ }^{2}=14.63 \mathrm{~dB}$ for the IFLF and LF filters respectively.

\section{Analysis and Comparison of OTA Nonidelities Effects}

The nonideal characteristic of OTAs will limit the performance of OTA-C filters. The nonideal model of the CMOS OTA includes the common-mode input capacitance $\mathrm{C}_{\mathrm{ic}}$, the differential-mode input capacitance $C_{i d}$, the output capacitance $C_{0}$, the output conductance $G_{0}$, and the frequency dependent transconductance $G_{m}(s)=g_{m}$ $/\left(1+s T_{p}\right)$, where $T_{p}=1 / \omega_{b}, \omega_{b}$ is the bandwidth of the OTA [6].

For frequency performance simulation, the IFLF and LF filters realise the same Butterworth characteristic in (6) with the chosen cut-off frequency of $1 \mathrm{MHz}$. The equal transconductance design is adopted with $\mathrm{g}_{\mathrm{m}}=30.14 \mu \mathrm{S}$. Then using $C_{j}=g_{m} \tau_{j}$, the normalised capacitances are computed. Frequency denormalisation gives the nominal circuit capacitances in Table 1. 
Table 1. Nominal circuit capacitances for fourth-order IFLF and LF filters.

\begin{tabular}{|c|c|c|c|c|}
\hline & $\mathrm{C}_{1}$ & $\mathrm{C}_{2}$ & $\mathrm{C}_{3}$ & $\mathrm{C}_{4}$ \\
\hline IFLF & $12.535 \mathrm{pF}$ & $6.268 \mathrm{pF}$ & $3.671 \mathrm{pF}$ & $1.836 \mathrm{pF}$ \\
\hline LF & $7.343 \mathrm{pF}$ & $7.566 \mathrm{pF}$ & $5.192 \mathrm{pF}$ & $1.836 \mathrm{pF}$ \\
\hline
\end{tabular}

Assume all OTAs have the same input impedance $Z_{\text {in }}$, output impedance $Z_{\text {out }}$ and frequency dependent transconductance $G_{m}(s)$. Thus, we can assign $C_{i c j}=C_{i c}$, $C_{i d j}=C_{i d}, G_{o j}=G_{o}, C_{o j}=C_{o}$ and $G_{m j}(s)=g_{m j} /\left(1+s T_{p j}\right)=$ $\mathrm{g}_{\mathrm{m}} /\left(1+\mathrm{s} \mathrm{T}_{\mathrm{p}}\right), \mathrm{j}=1,2,3,4$. The nonideal parameters in the macro-model of the CMOS OTA are assumed to be $\mathrm{C}_{\mathrm{id}}=$ $0.0385 \mathrm{pF}, \mathrm{C}_{\mathrm{ic}}=0.0502 \mathrm{pF}, \mathrm{G}_{\mathrm{o}}=113 \mathrm{nS}, \mathrm{C}_{\mathrm{o}}=0.52 \mathrm{pF}$ and $\mathrm{T}_{\mathrm{p}}=21.2 \mathrm{~ns}$.

From Fig.4, the effects of OTA nonidealities on the frequency performance of the two filters are quite obvious. For the IFLF filter, it is noticed that the nonideal response has significant deviation from the ideal response especially at the cut-off frequency and stop-band. For the LF filter, the nonideal performance is observed quite close to the ideal one. Thus, the LF filter is better than the IFLF filter in terms of frequency performance.

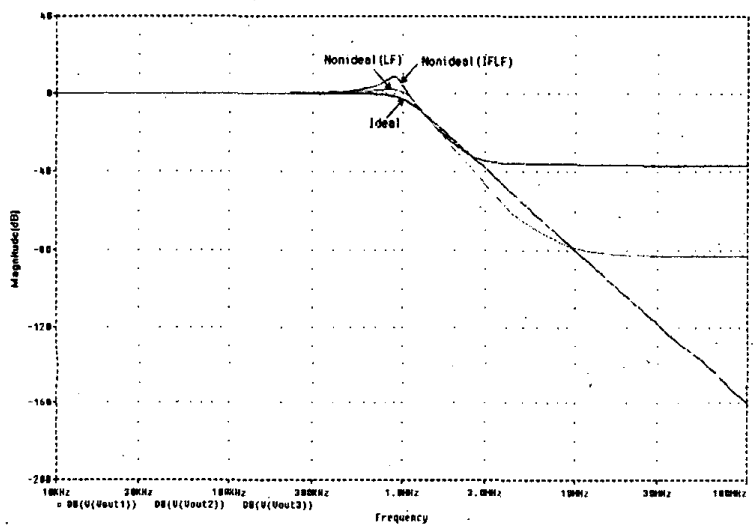

Fig.4. Simulated magnitude frequency responses of fourth-order IFLF and LF filters.

\section{Conclusions}

Comparisons of sensitivity, maximum input signal, noise, and OTA nonideality effects of the IFLF and LF allpole OTA-C filters have been presented for the fourthorder Butterworth low-pass characteristic with the cut-off frequency of $1 \mathrm{MHz}$. It has been shown that the LF structure has lower Schoeffler's multiparameter and worst case sensitivities than those of the IFLF structure. In terms of maximum input signal voltage magnitude, the LF filter has a slight higher value (difference by $0.07 \mathrm{~V}_{\mathrm{T}}$ ) than that of the IFLF filter. Over wide frequency range, the noise of the LF filter is much lower than that of the IFLF filter. In terms of the effects of OTA nonidealities on frequency performance, it has been shown that the LF structure is relatively less affected by these nonidealities than the IFLF structure. The overall conclusion is therefore that the LF structure is better than the IFLF structure.

Comparison simulations in the transistor level have confirmed the theoretical results. Clearly the results are suitable for balanced structure implementation of IFLF and LF filters [12], although they are obtained based on the single-ended structures.

\section{References}

[1] K. R. Laker, R. Schaumann, and M. S. Ghausi, "Multiple-loop feedback topologies for the design of low-sensitivity active filters", IEEE Trans. Circuits Syst., vol. 26, no. 1, pp. 1-21, Jan. 1979.

[2] T. Deliyannis, Y. Sun, and J. K Fidler, Continuostime active filter design, CRC press, Florida, USA, 1999.

[3] Y. Sun. and J. K. Fidler, "OTA-C realization of general high-order transfer functions", Electron. Lett., vol. 29, pp.1057-1058, 1993.

[4] Y. Sun and J. K. Fidler, "Minimum component multiple integrator loop feedback OTA-C all-pole filters", Proc. Midw. Symp. Circuits Syst., Lafayette, LA, USA, pp. 983-986, 1994.

[5] Y. Sun and J. K. Fidler, "Synthesis and performance analysis of universal minimum component integrator-based IFLF OTA-grounded capacitor filter', IEE Proc.-Circuits Devices Syst., vol. 143, no. 2, pp. 107-114, April 1996.

[6] Y. Sun and J. K. Fidler, "Structure generation and design of multiple loop feedback OTA-grounded capacitor filters", IEEE Trans. Circuits Syst., vol. 44, no. 1, pp. 1-11 Jan. 1997.

[7] Y. Sun, B. Jefferies, and J. Teng, "Universal thirdorder OTA-C filters", Int. J. Electronics, vol. 85, no. 5, pp. 597-609, 1998.

[8] D. H. Chiang and R. Schaumann, "A CMOS fullybalanced continuous-time IFLF filter design for read/write channels"', IEEE Proc. ISCAS, vol. 1, pp. 167-170, May 1996.

[9] G. Groenewold, "The design of high dynamic range continuos-time integratable bandpass filters", IEEE Trans. Circuits Syst., vol. 38, no. 8, pp. 838-852, Aug. 1991.

[10] F. N. Trofimenkoff, D. H. Treleaven, and L. T. Bruton, "Noise performance of RC-active quadratic filter sections", IEEE Trans. Circuit Theory, pp. 524-532, Sept. 1973.

[11] W. M. Snelgrove and A. S. Sedra, "Synthesis and analysis of state-space active filters using intermediate transfer function', IEEE Trans. Circuits Syst., vol. 33, no. 3, pp. 287-30, Mar. 1986.

[12] Y. Sun and J. K. Fidler, "Fully-balanced structures of continuous-time MLF OTA-C filters", Proc. IEEE Int. Conf. Electronics, Circuits and Systems, pp. $157-160$, Portugal 1998. 